\title{
Long Covid e sindrome post-Covid: nuove parole dalla pandemia
}

\author{
Kevin De Vecchis
}

PUBBLICATO: 30 SETTEMBRE 2021

$\mathrm{T}$ ra le parole nuove della pandemia da COVID-r9 (sul cui argomento si veda da ultimo Daniela Pietrini, La lingua infetta: l'italiano della pandemia, Roma, Treccani, 202I), alcune già trattate dal servizio di Consulenza linguistica dell'Accademia (come per es. contact-tracer e contact-tracing; droplet; lockdown) anche come rilanci (coronavirus; didattica a distanza; distanziamento sociale; link epidemiologico; quarantena; termoscanner), bisogna (purtroppo) registrare due nuovi ingressi: long Covid e sindrome post-Covid.

Si tratta di due termini che per il Devoto-Oli 2022 hanno lo stesso significato, ovvero la "condizione patologica riscontrata in pazienti che hanno superato l'infezione da Covid-r9, ma che continuano a manifestare alcuni sintomi (dolori muscolari, stanchezza cronica, sensazione di annebbiamento mentale, difficoltà di concentrarsi) anche a distanza di diversi mesi" (s.v. sindrome post-Covid; a long Covid vi è un semplice rinvio a sindrome post-Covid). Di diverso parere è l'Istituto Superiore di Sanità, che ha pubblicato un rapporto sulla malattia da COVID-I9 il I luglio 202I, dal titolo "Indicazioni ad interim sui principi di gestione del Long-COVID", per il quale long Covid è "una condizione clinica caratterizzata dal mancato ritorno da parte del paziente affetto da COVID-rg allo stato di salute precedente l'infezione acuta", che comprende sia "la malattia COVID-rg sintomatica persistente" sia "la sindrome post-COVID-r9", ovvero tutti quei "segni e sintomi che si sono sviluppati durante o dopo un'infezione compatibile con il COVID-r9, presenti per più di i2 settimane dopo l'evento acuto e non spiegabili con diagnosi alternative".

Da un punto di vista linguistico, long Covid è un composto inglese, formato dall'aggettivo long 'lungo' e da Covid, ed è entrato in italiano come locuzione invariabile di genere maschile (anche se non mancano casi al femminile, ma si veda quanto detto per il genere di COVID-Ig); sindrome post-Covid, invece, è una locuzione femminile italiana composta dal determinato sindrome e dal determinante postCovid, quest'ultimo già attestato in precedenza con il significato di 'periodo successivo alla pandemia da Covid-Ig' (Devoto-Oli 2022). Infine, bisogna segnalare anche la presenza, seppur minoritaria, della traduzione italiana di long Covid, ovvero Covid lungo, sulla cui origine e diffusione torneremo più avanti.

Essendo i termini di coniazione molto recente (come vedremo, si può risalire a maggio/giugno del 2020), non vi è ancora una stabilizzazione della loro forma scritta. Circolano, infatti, sulla rete e sui giornali numerose varianti grafiche. Riportiamo qui soltanto degli esempi, in base ad alcuni fattori di variazione (che combinati tra loro possono generare ulteriori forme):

- le iniziali dei costituenti possono essere tutte maiuscole:

Il Long Covid è una sindrome post-virale che può debilitare una persona sotto molti aspetti anche per parecchie settimane dopo la negativizzazione, e cioè dopo la guarigione e la conseguente eliminazione del virus dall'organismo. (Raffaella Gatta, Che cosa è il Long Covid? Quali sono i disturbi piu comuni??, articolo in www.marionegri.it, 28/6/202I) 
La Sindrome Post-Covid è una diretta conseguenza dell'infezione da SARS-CoV-2. Può comparire a negativizzazione avvenuta, con danni organici persistenti a polmoni, cuore, cervello e reni, causati probabilmente, in base agli studi ancora in corso, da risposte infiammatorie, microangiopatie trombotiche, tromboembolia venosa e carenza di ossigeno, anche in soggetti giovani e che hanno avuto sintomi da Coronavirus lievi. (Pagina di approfondimento in www.gvmnet.it)

- Covid può essere scritto interamente in maiuscolo e può essere accompagnato da "I9" o "-rg":

La possibilità che i sintomi durino nel tempo non sembra essere collegata a quanto si è stati male durante l'infezione. Può anche succedere che persone che hanno avuto una forma lieve di COVID-I9 possano sviluppare problemi a lungo termine. In generale, le donne al disotto dei 60 anni di età sembrano avere il doppio delle probabilità di manifestare il Long COVID rispetto agli uomini, successivamente il livello di rischio diventa simile tra i due sessi. (Pagina di approfondimento in www.isssalute.it, II/5/2021)

Nella maggior parte dei casi i sintomi scompaiono da soli. Se siete preoccupati per i sintomi della sindrome post COVID-rg o questi intralciano la vostra vita quotidiana, contattate il vostro medico e parlatene con lui per sapere come procedure. (Pagina di approfondimento in www.bag.admin.ch, 2I $/ 7 / 2 \mathrm{O} 2 \mathrm{I})$

- i costituenti possono essere separati da uno spazio, univerbati o collegati da un trattino:

Ma gli studi sul Long-Covid in fascia pediatrica che oggi abbiamo a disposizione hanno molti limiti: sono disomogenei, si basano spesso su risposte autoriferite o riferite dai genitori dei giovani pazienti e non sempre sono confermati da dati di laboratorio. (Tina Simoniello, Long-Covid nei bambini, dopo 12 settimane pochi strascichi. Ma la malattia lascia i segni anche da adulti, articolo in www.repubblica.it, 2I/9/2O2I)

Sul lungo termine esiste anche il longcovid ma quello, che é certificato non conta vero ? @LongCovidItalia (Tweet di @markocram8I del 2I/9/202I)

Lo vede quanto ci hanno messo i decessi a Marzo a passare da o a 20oo? Il rischio è la crescita esponenziale. Infine sembra preoccupante una strana sindrome postcovid che colpisce un Io\% di persone asintomatiche soprattutto di età media (Tweet di @antonio475317 del 6/10/2020)

Per quanto riguarda la storia di long Covid, covid lungo e sindrome post-Covid, se ripercorriamo le tappe che hanno portato all'individuazione della condizione patologica qui in esame, sarà possibile non solo ricostruire la diffusione mediatica che le voci hanno avuto, ma anche la loro origine.

Ai primi di maggio del 2020 un professore universitario di malattie infettive della Liverpool School of Tropical Medicine scrive un articolo in cui racconta la sua personale esperienza con la malattia da COVID-I9. Sul blog del British Medical Journal si legge dell'estrema difficoltà provata dall'uomo a guarire dalla malattia anche a distanza di settimane: "Health professionals, employers, partners, and people with the disease need to know that this illness can last for weeks, and the long tail is not some 'post-viral fatigue syndrome' - it is the disease" ['Gli operatori sanitari, i datori di lavoro, i colleghi e le persone che hanno contratto la malattia devono sapere che questa malattia può durare per settimane, e i postumi non sono qualche "sindrome da stanchezza post-virus', sono la malattia'] (Paul Garner, For 7 weeks I have been through a roller coaster of ill health, extreme emotions, and utter exhaustion, articolo in www.blogs.bmj.com, 5/5/2020). Tale condizione clinica, finora sconosciuta ai più e pertanto non denominata in modo ufficiale, nonostante le numerose testimonianze raccolte da Paul Garner, arriva anche all'attenzione della stampa italiana, sulle pagine della "Repubblica" (Elena Dusi, L'infettivologo: "Il 
$20 \%$ dei malati resta positivo al virus per 40 giorni", articolo in www.repubblica.it, 20/05/2020).

Lo stesso giorno, il 20 maggio 2020, una ricercatrice italiana dell'University College di Londra, Elisa Perego, usa la parola Long Covid (che si direbbe da lei stessa coniata) all'interno di un tweet in lingua inglese:

The \#LongCovid \#COVIDig is starting to be addressed on [sic] major newspapers in Italy too (Tweet di @elisaperego7 8 del 20/5/2020)

Il termine Long Covid compare cosi la prima volta in inglese sotto forma di hashtag per riunire le testimonianze di persone affette da questa condizione clinica (anche l'OED concorda con questa prima attestazione, s.v. long Covid).

All'interno degli scambi virtuali tra i diversi utenti, non solo italiani, che si sono prestati a diffondere notizie in merito, si può rintracciare anche il processo che ha portato alla creazione di un termine italiano. Da questo dialogo si evince anche come inizialmente ci si orientasse per covid persistente, lo stesso dello spagnolo, che però è poi stato scartato.

- Buongiorno Qui viviamo bene! Vous êtes franco-italienne, je pense. Vous pourriez nous dire si c'est ce hashtag \#covidpersistente que les italiens utilisent comme équivalent de notre \#apresJ2o et du \#CovidPersistente des espagnols? Nous cherchons ce \# depuis si longtemps! ['voi siete franco-italiani, penso. Potete dirci se è questo l'hashtag \#covidpersistente che gli italiani usano come equivalente del nostro \#apresJzo e dello spagnolo \#covidpersistente?'] (Tweet di @reverseyourmind del Io/6/2020)

- Bonjour, oui je parle les deux langues et vis à Venise. Malheureusement comme vous, j'ai longuement cherché sur le Twitter italophone mais il n'y a rien. du coup j'ai écrit \#covidpersistenteltalia mais il n' y a rien et aucun ou très peu d'intérêt pour la question ici ['Buongiorno, sì io parlo le due lingue e vivo a Venezia. Disgraziatamente come voi ho cercato a lungo su Twitter italofono ma non c'è nulla. Quindi ho scritto \#covidpersistenteItalia ma non ho trovato nulla e l'interesse qui per la questione è inesistente o molto scarso'] (Tweet di @quiviviamobene del Io/6/2020)

- Yes they are nice! Was wondering if a bit long. I thought of \#covidpersistente with the flag but is it good for Twitter? I like \#covidpersistenteitalia. Anche \#covidlungotermine. O \#covidlungo? [Si, sono carini! Mi chiedevo se fosse un po' troppo lungo. Ho pensato a \#covidpersistente con la bandiera ma funziona su Twitter? A me piace \#covidpersistenteitalia. Anche \#\#covidlungotermine. O \#covidlungo?'] (Tweet di @elisaperego78 del Io/6/2020)

A coniare il termine covid lungo, che è un evidente calco sull'inglese, ma che si potrebbe anche considerare riduzione di covid a (o di) lungo termine sembra si stata la stessa Elisa Perego: in suo tweet, pubblicato il 2I maggio, covidlungo compare accanto all'inglese LongCovid, al francese apres/9o, allo spagnolo covidpersistente e al tedesco MitCoronaLeben:

La distinzione tra dimessi/guariti e' [sic] fondamentale per capire il reale impatto di \#coronavirusitalia. Molti vanno incontro ad un processo di guarigione lungo, difficile\#covidlungo \#LongCovid \#MildCovid \#covidrinzo \#apresJ9o \#apresJ6o \#apresJ20 \#covidpersistente \#MitCoronaLeben (Tweet di @elisaperego78 dell'ı/6/2020)

Tuttavia, nonostante le buone premesse, la fortuna di long Covid oscura ben presto il traducente italiano, divenendo di fatto la forma preferita anche dagli utenti italiani. La prima attestazione di long Covid all'interno di un tweet in italiano compare pochi giorni dopo la creazione di Covid lungo, il i 8 giugno 2020: 
C’è il \#LongCovid e non è da sottovalutare (Tweet di @stefaniaconti del I8/6/2020)

Il termine ha una prima diffusione non solo come sostantivo, ma anche come aggettivo e aggettivo sostantivato dal significato di 'chi è affetto da long Covid':

In francia molti dei pazienti \#LongCovid erano giovani donne sane (Tweet di @stefaniaconti del $14 / 6 / 2020)$

Io ho sviluppato un sacco di igg ma i sintomi rintornano [sic] ciclicamente. Che tu sappia qualcuno dei \#longcovid ha provato qualche terapia? (Tweet di @depalop del 20/7/2020)

Diversamente da long Covid e Covid lungo che sembrano essere stati a tutti gli effetti coniati su Twitter, il termine sindrome post-Covid ha con ogni probabilità un'origine medica, messo poi in circolo dalla stampa. La prima attestazione scritta risale, infatti, al quotidiano di Piacenza "Libertà" e si deve al direttore dell'Unità Operativa Malattie Infettive dell'Ospedale di Piacenza, Mauro Codeluppi:

C’è chi ha ancora difficoltà respiratorie, chi accusa dolori al torace, chi si sente molto stanco: è la sindrome post Covid, una coda della malattia che per tanti pazienti ha già provocato grande sofferenza e a settimane di distanza dalla dimissione dall'ospedale o dalla guarigione a casa, impedisce ancora di tornare alle normali attività. (s.a., Sindrome post Covid, Codeluppi: "Tre mesi per capire le conseguenze, articolo in www.liberta.it, 25/6/2020; all'interno dell'articolo si può vedere il video dell'intervista)

Successivamente la locuzione compare su altri giornali, quali "Focus" e "Adnkronos". Su quest'ultimo si fa anche riferimento a uno studio scientifico di medici italiani, che però è stato scritto e pubblicato in inglese. (Angelo Carfi et al., Persistent Symptoms in Patients After Acute COVID-19, in "JAMA", 324, 2020, pp. 603-605)

Proprio perché la COVID-rg è una malattia nuova, non esistono ancora studi sugli effetti a lungo termine nei guariti all'infezione: nella fase dell'emergenza ci si è concentrati sul salvare la vita dei pazienti, e i primi a guarire, in Cina, sono usciti dagli ospedali solo pochi mesi fa. Tuttavia, un buon numero di persone sopravvissute a forme non necessariamente gravi di covid continua ad avvertire strascichi della malattia per settimane, addirittura mesi. Tanto che molti scienziati ritengono che la "sindrome post-covid" sia da considerarsi una famiglia di disturbi da seguire nel tempo, con fini di ricerca e riabilitativi. (Elisabetta Intini, Ė ora di parlare della sindrome post-covid, articolo in www.focus.it, $3 / 7 / 2020)$

Una stanchezza estrema che non passa, ma anche affanno o dolore alle articolazioni. Sono alcuni dei sintomi della cosiddetta sindrome post-Covid-19, che continua a tormentare la maggioranza dei 'reduci' dal coronavirus Sars-CoV-2 per settimane, se non addirittura per mesi dopo la guarigione. Una research letter appena pubblicata su 'Jama' da un gruppo di geriatri della Fondazione Policlinico Universitario Agostino Gemelli Irces e dell'Università Cattolica, campus di Roma, fa il punto della situazione e chiarisce quali sono i sintomi piu frequenti e persistenti. (s.a., Da stanchezza estrema a dolori, ecco sindrome post-Covid, articolo in www.adnkronos.com, $13 / 7 / 2020)$

È soltanto nell'agosto del 2020 che sui giornali, insieme all'italiano sindrome post-Covid, inizia a circolare anche il termine inglese long Covid (in un caso accompagnato anche da Covid a lungo termine):

In questi casi si parla di COVID a lungo termine, longcovid o sindrome post-COVID. «Le persone sono bloccate a letto e non sono in grado di andare a lavorare o prendersi cura dei propri figli», spiega Timothy Nicholson, neuropsichiatra del King's College di Londra, che sta studiando il fenomeno dopo aver sofferto lui stesso dei sintomi della COVID a lungo termine. (Angelo Romano, Perché é importante capire gli effetti persistenti e a lungo termine della COVID-19, articolo in www.valigiablu.it, 22/8/2020) 
Sempre più indizi e studi suggeriscono però che in una certa percentuale di individui la COVID-I9 abbia effetti assai più duraturi. In Italia è stato usato il termine "sindrome post-COVID-I9", mentre nei paesi anglosassoni si è diffusa l'espressione "long-haulers": le persone che si portano dietro i sintomi legati al coronavirus a lungo, a volte per mesi. [...] Oltre ai problemi fisici, queste persone devono fare i conti con le difficoltà del rientro al lavoro, e con la scarsa sensibilità pubblica nei confronti della cosiddetta "long COVID", sconosciuta ai più. (s.a., Quelli che non guariscono dalla COVID-19, articolo su www.ilpost.it, $26 / 8 / 2020)$

Stanchezza, debolezza, fiato corto, eritemi, perdita di memoria, ansia e dolori muscolari sono alcuni dei sintomi che accomunano le persone che soffrono di quella che in Italia qualcuno inizia chiamare "sindrome post-Covid". All'estero vengono chiamati "Long Covid" o "Long Haulers" (letteralmente "trasportatori a lunga distanza") e sono persone ufficialmente guarite e negative al tampone che però hanno sintomi persistenti e disturbi che durano da più di tre mesi. (Silvia Turin, Long Covid: chi è negativo, ma non è mai guarito (e ha sintomi da mesi), articolo su www.corriere.it, 27/8/2020)

Ad oggi la forma che sembra aver conquistato lo spazio maggiore è senz'altro long Covid. Se misuriamo la diffusione delle tre parole concorrenti nelle pagine in italiano di Google (dati aggiornati al I9/o9/202I), otteniamo: per long Covid 251.00o risultati (r.) e per long-Covid 526.00o r; per sindrome post Covid 57.000 r. e per sindrome post-Covid 56.600 r., per Covid lungo 7.0oo r. (poco affidabili in quanto lungo potrebbe essere anche preposizione, es. casi di Covid lungo il 2021) e per Covid-lungo 6.480 r.; possiamo aggiungere: Covid a lungo termine con 4I.6oo r. e Covid di lungo termine con 3.480 r. Cosi anche sull'archivio della "Repubblica": long Covid e long-Covid registrano rispettivamente 42 r. e 3 r., sindrome post-Covid e sindrome post Covid 6 r. e 5 r., Covid lungo 2 r.; su quello del "Corriere della sera": long Covid ha 20 r. e long-Covid 4 r., sindrome post covid e sindrome post-Covid 2 r. ciascuno, Covid lungo 2 r.; sul sito invece della "Stampa" si hanno: per long Covid 27 r. (nessun risultato per long-Covid), per sindrome post-Covid 2 r. (nessun risultato per sindrome post Covid), per Covid lungo I r. e per Covid a lungo termine $\mathrm{I} r$.

La fortuna di long Covid si deve senz'altro al prestigio della lingua inglese e all'ufficializzazione da parte di alcune istituzioni sanitarie del termine. Esso infatti compare a dicembre 2020 nelle linee guida ufficiali per gestire il "long COVID" del NICE (National Institute for Health and Care Excellence) e all'interno di una normativa sulla malattia della WHO (World Health Organization). Non ci sembra, invece, che il Ministero della Salute italiano abbia redatto documenti o comunicazioni ufficiali in merito (gli unici documenti rintracciati sono il Progetto "Piano Operativo Nazionale per la Previsione e la prevenzione degli Effetti delle ondate di calore sulla Salute" del I7/5/202I, dove si leggono i termini "sindrome cronica" e, tra parentesi, "(long-term COVID-I9)", e un articolo online dell'ıг/6/2021, dove si utilizza "sindrome post Covid", sempre al fianco di "long-term COVID-ro"). Anche il rapporto dell'ISS riportato all'inizio ufficializza difatti il termine long Covid.

Solo il tempo (e anche in base alla direzione che verrà presa dalle autorità sanitarie ministeriali e paraministeriali) potrà dirci di più sullo sviluppo delle tre parole, anche se il destino sembra ormai segnato: mentre le altre lingue principali europee sembrano aver virato verso soluzioni 'autoctone', l'italiano si è adeguato, ancora una volta, al modello inglese. Per ora possiamo soltanto riflettere sulla portata linguistica della pandemia, che da ormai più di un anno irrompe violentemente all'interno della nostra vita con ripercussioni evidenti anche sul piano lessicale. Da questo affluente continuiamo a pescare parole inglesi, che in ambito medico e socio-sanitario dimostrano la loro dominanza. Il nostro augurio è che lo scorrere della pandemia possa un giorno arrestarsi, lasciando tutte queste parole (italiane o straniere non importa) sul fondo. 


\section{Cita come:}

Kevin De Vecchis, Long Covid e sindrome post-Covid: nuove parole dalla pandemia, "Italiano digitale", XVIII, 2021/3 (luglio-settembre)

DOI: $10.35948 / 2532-9006 / 2021.11612$

Copyright 2021 Accademia della Crusca

Pubblicato con licenza creative commons CC BY-NC-ND 